\section{0-347 LOW BACK PAIN OCCURRENCE AND EVOLUTION IN DIFFERENT WORK SITUATIONS DURING COVID-19 EPIDEMIC LOCKDOWN FROM 17 MARCH TO 10 MAY 2020, IN METROPOLITAN FRANCE}

${ }^{1}$ Emilie Chazelle, Natacha Fouquet, Christine Chan Chee. 'Santé Publique France, France

\subsection{6/OEM-2021-EPI.31}

Introduction The work organization of most French workers was significantly modified (extended implementation of teleworking, work interruptions...) during the first mandatory lockdown set up to fight the spread of the COVID-19 pandemic, from 17 March to 10 May 2020.

Objectives The aim of the study was to estimate the effects of the changes in the work organization due to lockdown on low back pain onset and its evolution.

Methods Workers were retrospectively interviewed online during three waves of the Coviprev study (access panel in the general population) between 8 June and 8 July 2020 about their work situation during lockdown and their low back symptoms before and at the end of the lockdown.

Results The rate of low back pain onset in workers who did not have any low back symptoms prior to the lockdown $(n=2,113)$ was $10.4 \%$ [9.1-10.7]. The work situation during the lockdown was associated with significantly increased risks of low back pain in people who began teleworking due to lockdown, and in those who continued to work outside home at a higher pace than usual (compared to working outside home as usual; odds ratio of 2.81 [1.77-4.46] and 2.76 [1.50-5.09], respectively). The work situation during lockdown was also significantly associated with the evolution of low back pain in workers who already suffered from it before the lockdown ( $\mathrm{n}=1,111,34.3 \%$ [32.7-36.0]) with an increased risk of worsening for those who worked outside the home at a higher pace than usual (odds ratio 3.39 [1.38-8.31]) and a higher probability of improvement for those who usually worked from home before the lockdown (odds ratio 1.86 [1.00-3.43]).

Conclusion While the Covid-19 epidemic and teleworking take hold, measures should be implemented in order to prevent an increase in the burden of low back pain in workers.

\section{0-446 OCCUPATIONAL RISK FACTORS FOR SARS-COV-2 INFECTION AND COVID-19: RESULTS FROM THE COVICAT COHORT STUDY IN CATALONIA, SPAIN.}

1Kurt Straif, Ana Espinosa, Gemma Castaño Vinyals, Marianna Karachaliou, Miguel Angel Alba Hidalgo, Kyriaki Papantoniou, Judith Garcia-Aymerich, Rafael de Cid, Ximena Goldberg, Manolis Kogevinas. 'ISGlobal and Boston College, France

\subsection{6/OEM-2021-EPI.32}

Introduction During the first lockdown in Spain (March-June, 2020) essential workers may have been at increased risk of coronavirus disease 2019 (COVID-19) via occupational exposure. Results from published studies are heterogeneous.

Methods Ongoing population-based cohort studies from Catalonia were pooled to form the COVICAT study. A random sub-population donated a blood sample (May-July, 2020) for validated multiplex serology testing. Occupational analyses were restricted to working age (18-65 years). Participants responded to a web-based or telephone survey including questions on socio-demographics, pre-pandemic health, behavioural and environmental risk factors. Occupational questions covered mode of work (e.g. telework), job title, availability of personal protective equipment (PPE), and mode of commuting. Job titles were coded by an occupational hygienist to the Spanish CNO-11 and cross-walked to ISCO-08. COVID-19 cases were defined by symptoms or hospitalisation and SARS CoV-2 seropositivity based on immune responses to 15 isotype-antigen combinations (serology sub-cohort). Logistic regression models were built for type of work, job titles and job-exposure matrix (JEM), covering several dimensions and levels of SARS-CoV-2 transmission probabilities, and adjusted for age, sex, education, deprivation index, population density and survey type.

Results This analysis included 8,582 participants, of which 3,599 were tested for SARS-CoV-2 antibodies, median (SD) age 53.7 (6.3) years, $59.9 \%$ were women. The relative risk for COVID-19 for work in the usual workplace compared to telework was 1.87 (95\% CI: 1.44, 2.42), and 1.44 (95\% CI: $1.09,1.90)$ among the serology study. The relative risk for nurses who worked in their usual workplace was 4.57 (95\% CI: $3.12,6.7)$. Detailed results by job title, JEM, availability of PPE and commuting mode will be presented.

Conclusions This study has several strengths, including random serology testing and individual-level exposure data. Detailed results may support extended legal definitions of COVID-19 as a recognized occupational disease.

\section{COVID19-2}

\section{0-151 ARE HEALTHCARE WORKERS USING PERSONAL PROTECTIVE EQUIPMENT DURING THE COVID-19 PANDEMIC?}

${ }^{1}$ Christine Cramer, Karoline Kærgaard Hansen, Esben Meulengracht Flachs, Martin Byskov Kinnerup, Kent Jacob Nielsen, Ole Carstensen, Jesper Medom Vestergaard, Jens Peter Ellekilde Bonde, Karin Biering, Else Toft Würtz, Annett Dalbøge, Ane Marie Thulstrup, Mette Lausten Hansen, Anne Mette Lund Würtz, Vivi Schlünssen, Henrik Albert Kolstad. 'Department of Public Health, Work, Environment and Health, Danish Ramazzini Centre, Aarhus University, Aarhus, Denmark

\subsection{6/OEM-2021-EPI.33}

Introduction Personal protective equipment (PPE) can prevent transmission of COVID-19; however, proper use requires time, experience, training and sufficient supplies of PPE.

Objective The aim of the overall study is to investigate the risk of COVID-19 among Danish healthcare workers. Here, the availability and use of PPE among the Danish healthcare workers during the pandemic is investigated.

Methods Healthcare workers employed at the Central Denmark Region $(32,413)$ and the Capital Region of Denmark $(38,807)$ on April 1, 2020 were invited to respond to daily questionnaires from April 24, 2020, until June 30, 2020 that addressed work tasks, contact with COVID-19 patients, use of PPE and breach of PPE. On November 17, 2020, 26,092 healthcare workers from the Central Denmark Region and on December 15, 2020, 9,664 from the Capitol Region of Denmark were invited to a second round of daily questionnaires, and were followed until April and March 30, 2021, respectively.

Results In total, 12,328 (38.0\%) and 9,664 (24.4\%) healthcare workers participated in the first round of the study, respectively in the Central Denmark Region and the Capital Region of Denmark. One fifth $(20.3 \%$ and $20.6 \%$ 